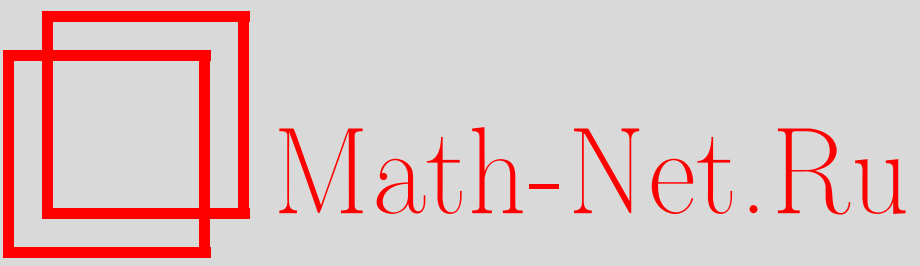

Х. фон Вайцзеккер, О. Г. Смолянов, Формулы с логарифмическими производными мер, связанные с квантованием бесконечномерных гамильтоновых систем, УМH, 1996, том 51, выпуск 2, 149-150

DOI: https://doi.org/10.4213/rm950

Использование Общероссийского математического портала Math-Net.Ru подразумевает, что вы прочитали и согласны с пользовательским соглашением

http://www. mathnet.ru/rus/agreement

Параметры загрузки:

IP : 54.197 .217 .227

26 апреля 2023 г., 15:52:54 


\title{
ФОРМУЛЫ С ЛОГАРИФМИЧЕСКИМИ ПРОИЗВОДНЫМИ МЕР, СВЯЗАННЫЕ С КВАНТОВАНИЕМ БЕСКОНЕЧНОМЕРНЫХ ГАМИЛЬТОНОВЫХ СИСТЕМ
}

\author{
Х. ФОН ВАЙЦЗЕККЕР, О.Г. СМОЛЯНОВ
}

Формулы, содержащие логарифмические производные (гладких) мер на бесконечномерных пространствах, возникают в самых различных ситуациях. Так, логарифмические производные меры входят в уравнение Шрёдингера в пространстве, состоящем из функций, квадратично интегрируемых по этой мере, что позволяет очень просто описать процедуру (канонического) квантования бесконечномерных гамильтоновых систем с линейным фазовым пространством. Далее, задача восстановления меры по ее логарифмической производной (впервые поставленная независимо от каких-либо приложений в 1971 году [1]) может быть равносильна задаче нахождения "основного состояния" (понимаемого как некоторая мера) для бесконечномерного уравнения Шрёдингера или задаче нахождения инвариантной меры для стохастического дифференциального уравнения, или задаче восстановления гиббсовской меры по ее спецификации. Логарифмические производные мер появляются в формулах Камерона-Мартина-Гирсанова-Маруямы-Реймера и в их обобщениях, относящихся к произвольным гладким мерам; они же позволяют связать эти формулы с формулой Фейнмана-Каца.

1. Логарифмические производные, логарифмические плотности, обобщенные плотности гладких мер. Далее все векторные пространства предполагаются вещественными. Для каждого локально выпуклого пространства (ЛВП) $E$ символ $\mathscr{B}(E)$ обозначает $\sigma$-алгебру его борелевских подмножеств, а символ $\mathfrak{M}(E)$ - векторное пространство счетноаддитивных вещественнозначных мер на $\mathscr{B}(E)$. Если $h$ - векторное поле на ЛВП $E$ (т.е. отображение $E$ в $E$ ), то через $T_{h}$ обозначается отображение $E$ в $E$, определяемое так: $T_{h}(x)=x+h(x)$. Если еще $\nu \in \mathfrak{M}(E)$, то через $\nu_{h}$ обозначается образ $\nu$ при отображении $T_{h}$. Мера $\nu$ назьвается дифференцируемой (по Фомину) вдоль векторного поля $h$, если функция $f: \mathbb{R}^{1} \rightarrow \mathfrak{M}(E)$, $t \mapsto \nu_{t h}$, дифференцируема в нуле при наделении пространства $\mathfrak{M}(E)$ топологией сходимости на каждом множестве из $\mathscr{B}(E)$; при этом мера $-f^{\prime}(0)$, обозначаемая символом $d_{h} \nu$, называется производной (Фомина) меры $\nu$ вдоль векторного поля $h$ (cp. [2]). Оказывается, что $d_{h} \nu$ абсолютно непрерывна относительно $\nu$ (ср. [1] и [2]), так что $d_{h} \nu=\beta_{h}^{\nu} \cdot \nu$; функция $\beta_{h}^{\nu}$ назьвается логарифмической производной меры $\nu$ вдоль векторного поля $h$. Если, в частности, $h(x)=k$ для всех $x \in E$, то вместо символа $\beta_{h}^{\nu}$ используется символ $\beta^{\nu}(h, \cdot)$. Отметим, что если $D(\nu)=\left\{k \in E: \exists \beta^{\nu}(k, \cdot)\right\}$, то отображение $k \mapsto \beta^{\nu}(k, \cdot), D(\nu) \rightarrow L_{1}(E, \nu)$, линейно [1]. Если $H$ - гильбертово подпространство пространства $E, H \subset D(\nu)$ и для каждого $x \in E h(x) \in H$ (а также выполнены некоторые условия регулярности), то $\beta_{h}^{\nu}(x)=\beta^{\nu}(h(x), x)+\operatorname{tr} h^{\prime}(x), x \in E$ (см. [2] и имеющиеся там ссылки).

ПредЛоЖение 1. Если отображение $\beta^{\nu}: H \times H \rightarrow \mathbb{R}^{1},(h, x) \mapsto \beta^{\nu}(h, x)$, непрерьвно дифференцируемо, то существует функция $\sigma_{\nu}: H \rightarrow \mathbb{R}^{1}$, для которой при всех $h, x \in H$ справедливо равенство $\sigma_{\nu}^{\prime}(x)=\beta^{\nu}(h, x)$.

Функцию $\sigma_{\nu}$, определенную на $H$, естественно назвать логарифмической плотностью меры $\nu$. Функцию $H \ni x \mapsto \exp \sigma_{\nu}(x)$ можно назвать обобщенной плотностью меры $\nu$ (cp. [3]).

ПРеДЛОЖЕнИЕ 2. Если $\Lambda_{\nu}$ - непрерывная вещественная функция на $H \times E(H \subset D(\nu))$, сужение которой на $H \times H$ совпадает с функиией $(h, x) \mapsto \sigma_{\nu}(x+h)-\sigma_{\nu}(x)$, то $\Lambda_{\nu}(h, x)=\int_{0}^{1} \beta^{\nu}\left(F_{1}^{\prime}(\tau, x), F(\tau, x)\right) d \tau$, әде $F$ - отображсение $\mathbb{R}^{1} \times E \rightarrow E$ (удовлетворяющее некоторым условиям регулярности [4]), такое, что $F(0, x)=x, F(1, x)=x+h$.

Второй автор пользовался поддержкой Российского фонда фундаментальных исследований (грант № 93-011-1693) и Международного научного фонда (грант № ND 8000). 
2. Квантование. Пусть $Q$ и $P$ - два экземпляра гилбертова пространства со скалярным произведением $(\cdot, \cdot), E=Q \times P$ и $\mathscr{H}-($ вешественная) функция на $E$, определяемая равенством $\mathscr{H}(q, p)=(A p, p)+V(q)$, где $A$ - самосопряженный положительно определенный ядерный оператор в $P$. Далее, пусть $\nu$ - бесконечно дифференцируемая по гильбертову подпространству $H$ мера из $\mathfrak{M}(Q), \psi_{\nu}$ - обобщенная плотность меры $\nu$. Меру $\eta$ с обобщенной плотностью $\psi_{\nu}^{2}$ (если такая мера существует) естественно назвать квадратом меры $\nu$, так что можно написать $\eta=\nu^{2}$. Положим $G=L_{2}\left(Q, \nu^{2}\right)$ и определим линейный оператор $\widehat{\mathscr{H}}_{\nu \text { в }} G$ равенством $\left(\widehat{\mathscr{H}}_{\nu} g\right) \nu=$ $\widehat{\mathscr{H}}(g \nu)$, где $\widehat{\mathscr{H}}$ - дифференциальный оператор в $\mathfrak{M}(E)$ с символом $\mathscr{H}$.

TeOpema 1. Ecлu $g \in \operatorname{dom} \widehat{\mathscr{H}}_{\nu}, m o$

$$
\left(\widehat{\mathscr{H}}_{\nu} g\right)(x)=\operatorname{tr}\left(A\left(g^{\prime \prime}(x)+\beta^{\nu}(\cdot, x) \otimes g^{\prime}(x)\right)+\frac{d(\widehat{\mathscr{H}} \nu)}{d \nu}(x) \cdot g(x) .\right.
$$

\section{3. Сопряженные операторы, инвариантные меры и т. д.}

ПрЕДЛОЖЕниЕ 3. Если $\widehat{\mathscr{H}} \nu=0$, mo $\left(\widehat{\mathscr{H}}_{\nu} g\right)(x)=\operatorname{tr}\left(A g^{\prime \prime}(x)+\beta^{\nu}(\cdot, x) \otimes g^{\prime}(x)\right)$.

Далее предполагается, что $\nu$ - основное состояние для оператора $\widehat{\mathscr{H}}$, т.е. $\widehat{\mathscr{H}} \nu=0$.

ПрЕДЛОЖЕниЕ 4. Если $\widehat{\mathscr{H}}_{\nu}^{*}$ - линейный оператор (не всюду определенньй) в пространстве $\mathfrak{M}(Q)$, сопряженный $к \widehat{\mathscr{H}}_{\nu}$ относительно естественной двойственносmu между $L_{1}(Q, \nu) \cap \operatorname{dom} \widehat{\mathscr{H}}_{\nu}$ u $\mathfrak{M}(Q)$, mo $\widehat{\mathscr{H}}_{\nu}^{*} \mu=\operatorname{tr} A\left(\mu^{\prime \prime}+\left(\beta^{\nu}\right)_{2}^{\prime}(\cdot, \cdot) \mu+\beta^{\nu}(\cdot, \cdot) \otimes\right.$ $\left.\beta^{\mu}(\cdot, \cdot) \mu\right)=\operatorname{tr} A D^{*} D \mu$, где $D^{*}$ - оператор, сопряженный (относительно двойственности пространств функций на $Q$, определяемой мерой $\nu) \kappa$ оператору дифференцирования D.

ТЕорема 2. Следующие соотношения и утверждения равносильны: (1) $\widehat{\mathscr{H}} \nu_{0}=0$; (2) $\widehat{\mathscr{H}}_{\nu}^{*} \nu_{0}=0$; (3) $\nu_{0}^{2}-$ инвариантная мера для стохастического дифференциального уравнения $d x=\beta^{\nu}(\cdot, x) d t+d w$; (4) $\nu_{0}-$ мера с логарифмической производной $\beta^{\nu}(\cdot, \cdot)$.

ЗАмечАние 1 . Операторы $\mathscr{H}_{\nu}$ и $\mathscr{H}_{\nu}^{*}$ входят, соответственно, в прямое и обратное уравнения Колмогорова, соответствуюшие уравнению из теоремы 2.

4. Формула Фейнмана-Каца. Если $\nu$ - вероятностная мера на $E$ с плотным (в $E)$ подпространством $H$ дифференцируемости, $\psi_{\nu}$ - ее обобщенная плотность и $g$ - (неотрицательная) функция на $E$, то вероятностная мера $\eta$ с обобщенной плотностью $g \cdot \psi_{\nu}$ (если она существует) обозначается символом $g \cdot \nu$.

Теорема 3. Справедливо равенство $\exp \left(-t \widehat{\mathscr{H}}_{\nu} g\right)(x)=\int \exp \left(\int_{0}^{t} V(x+b(\tau)) d \tau\right) \times$ $g(x+b(t)) \Lambda_{\nu}(b(t), x) w(d b)$, əде $w$ - мера Винера на пространстве обращающихся в нуле в нуль непрерывных функций на $[0, t]$, принимающих значения в $Q$, переходная вероятность которой порождается коррелячионным оператором $A($ ср. [5]).

\section{СПИСОК ЛИТЕРАТУРЫ}

[1] Авербух В. И., Смолянов О. Г., Фомин С. В. // Тр. ММО. 1971. Т. 24. С. 133-174. [2] Smolyanov O. G., von Weizsäcker H. // J. Funct. Anal. 1993. V. 188. № 2. P. 455-476. [3] Кириллов А. И. // УМН. 1994. Т. 49. № 3. [4] Smolyanov O. G., von Weizsäcker H. // C. R. A. Sci. Paris. Ser. 1. 1995. V. 321. P. 103-108. [5] Simon B. Functional integration and quantum physics. New York: Academic Press, 1979. 